Discussion on Paper No. 6758*

\title{
Wind-excited oscillations of structures
}

\author{
by \\ C. Scruton, B.Sc., A.F.I.A.A. \\ and
}

A. R. Flint, B.Sc., Ph.D., A.M.I.C.E.

Mr G. Roberts (Messrs Freeman, Fox \& Partners) remarked on a point which had always seemed a little strange. A smooth cylindrical object was prevented from oscillating in the wind by the addition of helical fins, whereas an object of helical construction, such as a stranded rope, had smooth tape wrapped round it to stop it oscillating.

85. The Forth tower oscillated in an apparently alarming and dangerous manner, but it was not at all dangerous. The connexions between the sections of the tower were made with longitudinal high-tensile bolts of waisted section about $3 \mathrm{ft}$ long, which when overstressed could stretch several inches without any danger of fracture.

86. It was realized in the design stages that oscillation might occur, but at that time there was no means of estimating the amplitude that would build up. It was known that if the tower did oscillate it could not be catastrophic because at a certain stage the bolts in the joints would be overstressed and the joints would open, so that the sections of the tower would rock on each other like a series of bricks unconnected, the opening and closing providing sufficient damping to reduce the oscillations or prevent them increasing.

87. That had in fact happened, but not until the double amplitude had built up to about $7 \mathrm{ft}$, which made the erectors seasick. The Contractors could not resist the temptation to keep on tightening up the bolts so that the stretching was progressive and there was a limit to their extensibility.

88. Something had to be devised quickly to stop the oscillation. They wanted the tower to remain substantially vertical, and did not want to pull it back, which would have occurred if they had attached heavy cables and applied a large force. A bridle damping service was therefore devised.

89. The device (Fig. 16) consisted of two lengths of 2-in. dia. rope tied down to the ground at 100 -ft intervals to give it some inherent stiffness, so that it would move the weight attached to the end at an amplitude of about 9 in. Fig. 17 showed the weight, which was a floating bridge section filled with concrete. A rope was attached to it, passing over a pulley and attached to the bridle. The weight was resting on a pair of steel rails sloping at $45^{\circ}$, and any movement of the tower top would pull it up or down. It was calculated that the loss of energy due to the friction of weight on the rails would be sufficient to damp the oscillation. This did not exert too much force on the tower top, and the damping was sufficient to stop the oscillations when the amplitude reached about 9 in.

* Proc. Instn civ. Engrs, vol. 27, April 1964, pp. 673-702. 
The Chairman, Mr H. Shirley-Smith, speaking for the Contractors and wishing to remove the impression that they overtightened any bolts, explained that the bolts were tightened exactly to the degree called for in the Erection Memorandum, i.e. they were hand tight.

91. Regarding the aerodynamic oscillation of the towers, the erectors had not only been seasick but had been thrown about and bruised and it had been necessary to bring them down every time the movement started. The sway of $7 \mathrm{ft} 6 \mathrm{in}$. had a period of $4 \frac{1}{2} \mathrm{~s}$ and in gentle winds it used to build up and die down about every three minutes. The lifts could not be worked during this time and the cranes had to be tied up, resulting in considerable delay. Oscillation occurred only in very light and steady winds of $20-25 \mathrm{mile} / \mathrm{h}$. In a wind of $70-80 \mathrm{mile} / \mathrm{h}$ so much turbulence and eddies were set up that the tower merely shuddered a few inches.

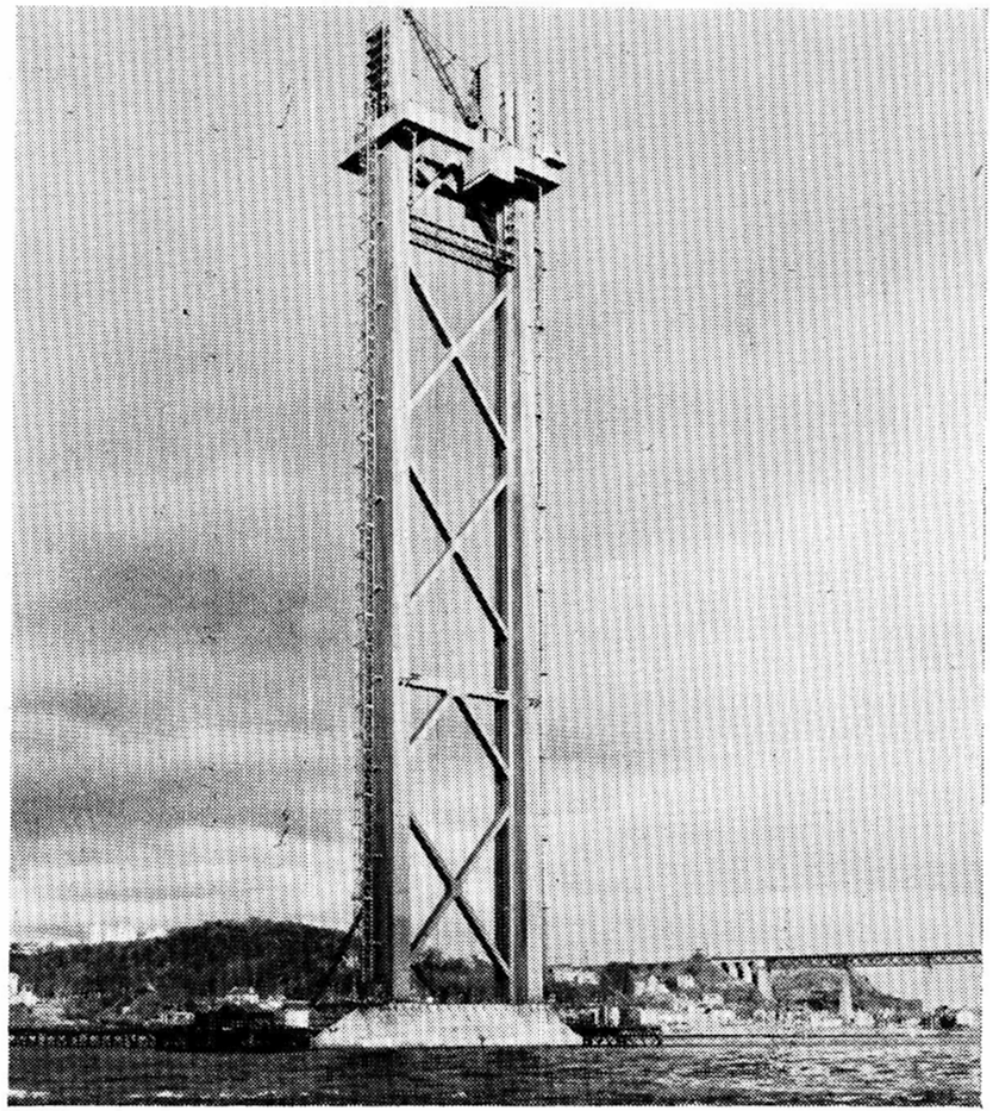

FIG. 16 
92. He wished to correct the Paper on one point--both towers behaved, or would have behaved, in the same way, but the phenomenon was observed in the North tower because that was the first one to be built.

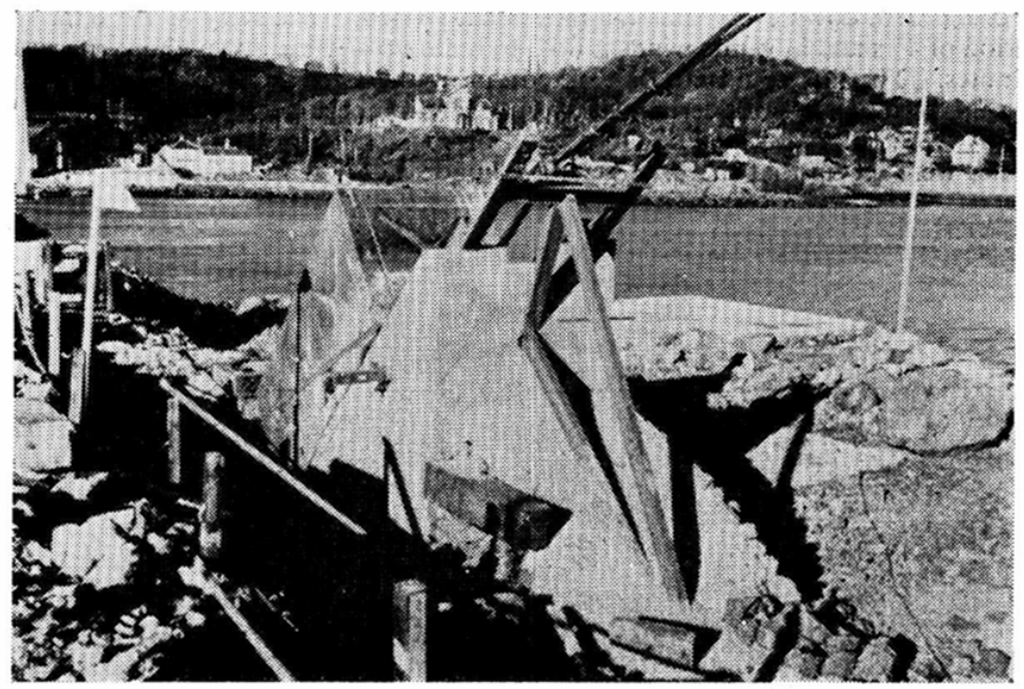

FIG. 17

93. When it had reached a height of $400 \mathrm{ft}$ a sway of more than $3 \mathrm{ft}$ occurred at the top (see Fig. 18). It was then that he had to make the very difficult decision whether to continue erection, which would obviously increase the sway, or not. The decision had been made to move the climbing structure up and to continue building, and he was glad to say that, after the very efficient damping service subsequently designed by Mr Gilbert Roberts had been installed, the sway at the tower top never again reached any significance.

94. Concerning possible aerodynamic action on the deck, the Chairman said that it had been intended to build out the full width of the two 24-ft wide steel battledecks in the main span and also the cantilevered cycle tracks and footpaths in the first pass, working outwards from the towers.

95. Tests on small scale models of the deck steelwork, carried out under the direction of Mr Scruton at the request of the Contractors, showed that if this had been attempted the steelwork would have been unstable in gale force winds. Its vulnerability would have been increased by the fact that the inevitable temporary distortion of the steelwork at this stage (see Fig. 19) necessitated leaving the lower chord connexions open, and this deprived the deck of much of its torsional rigidity.

96. Further wind tunnel tests were made on a number of possible methods of erection. These showed that if the suspended steelwork was erected in two stagesfirst leaving out the centre panels of the roadway, so that an open slot $25 \mathrm{ft}$ wide was left right along the centre line of the bridge, and omitting the cantilevered footways and cycle tracks until junction had been effected at mid-span-it would be stable in 


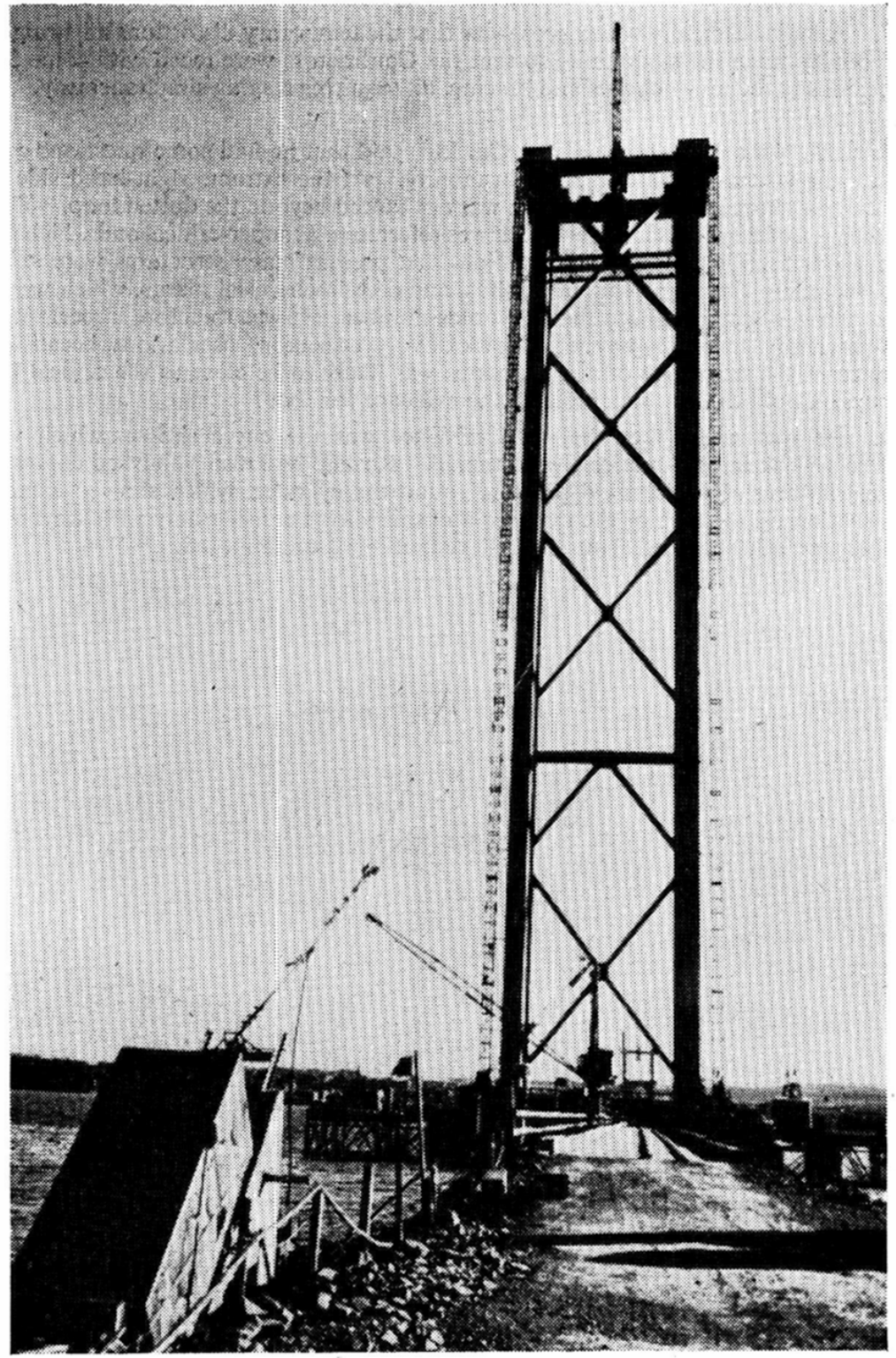

Fig. 18: ERection OF NORTH TOWER NEARING COMPLETION 
winds of up to $100 \mathrm{mile} / \mathrm{h}$ or more (see Fig. 20). This method was followed at site and the remainder of the steelwork was subsequently assembled by gantries working out from the towers in the second pass (see Fig. 21).

97. A major difficulty which arose was that the temporary distortions all straightened out at much the same time, so that the Contractors were faced with some 300 connexions in the lower chords and bracing, all requiring staging simultaneously.

Mr L. G. Wills (Director, Holst \& Co. Ltd) said that he had some questions concerning the vortex-shedding type of instability. At the extreme right-hand side of Fig. 2 was a short length of line which was not dotted beyond the dotted loop. That was the area of Reynolds number which was referred to as super-critical and which ran from about 5 million to about 15 million. At present many structures were being built, including very large chimneys, silos, and certain chemical plants, which would be sensitive to wind at about that Reynolds number. It was therefore important to be sure whether there was any serious risk of vortex-shedding oscillations, because in many cases the frequency of the structures was likely to be close to the critical frequency. Could the Authors indicate the evidence for that ?

99. He was aware that the main evidence was the curve shown, which was Roshko's work in an American wind tunnel. Roshko had also published curves of the distribution of radial wind pressure round the cylinder, which showed a fairly high suction on the side of the cylinder and this varied considerably with Reynolds number and other factors, from, he believed, about 3 to about $0 \cdot 8$.

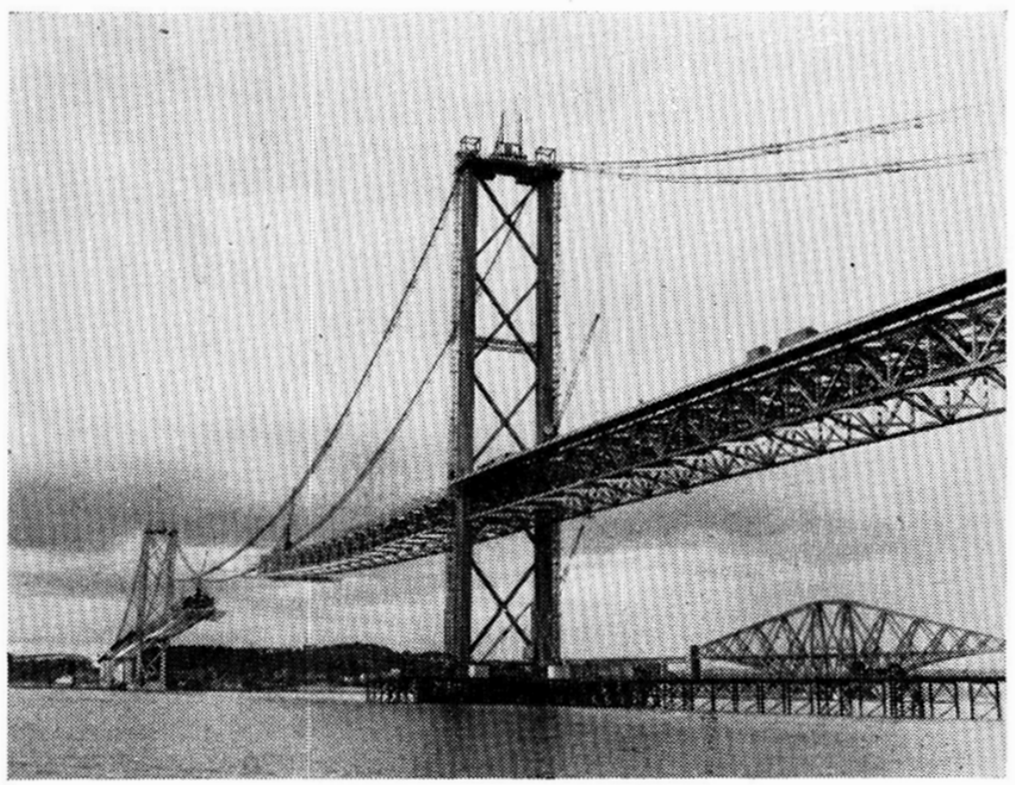

Fig. 19: TeMPORARY DISTORTION OF STEELWORK DURING ERECTION OF DECK IN MAIN SPAN 
100. The French Government distribution was the oldest and showed a maximum of 0.8 for the suction on the side. It was doubtless a practical rule, drawn up for structures which would have a high Reynolds number and which were probably quite short when the ratio of length to diameter was not very great. It therefore seemed that side suction and liability to vortex shedding might vary together, since they were associated with the same part of the air flow. Were there any reports of practical trouble with real structures in that super-critical range?

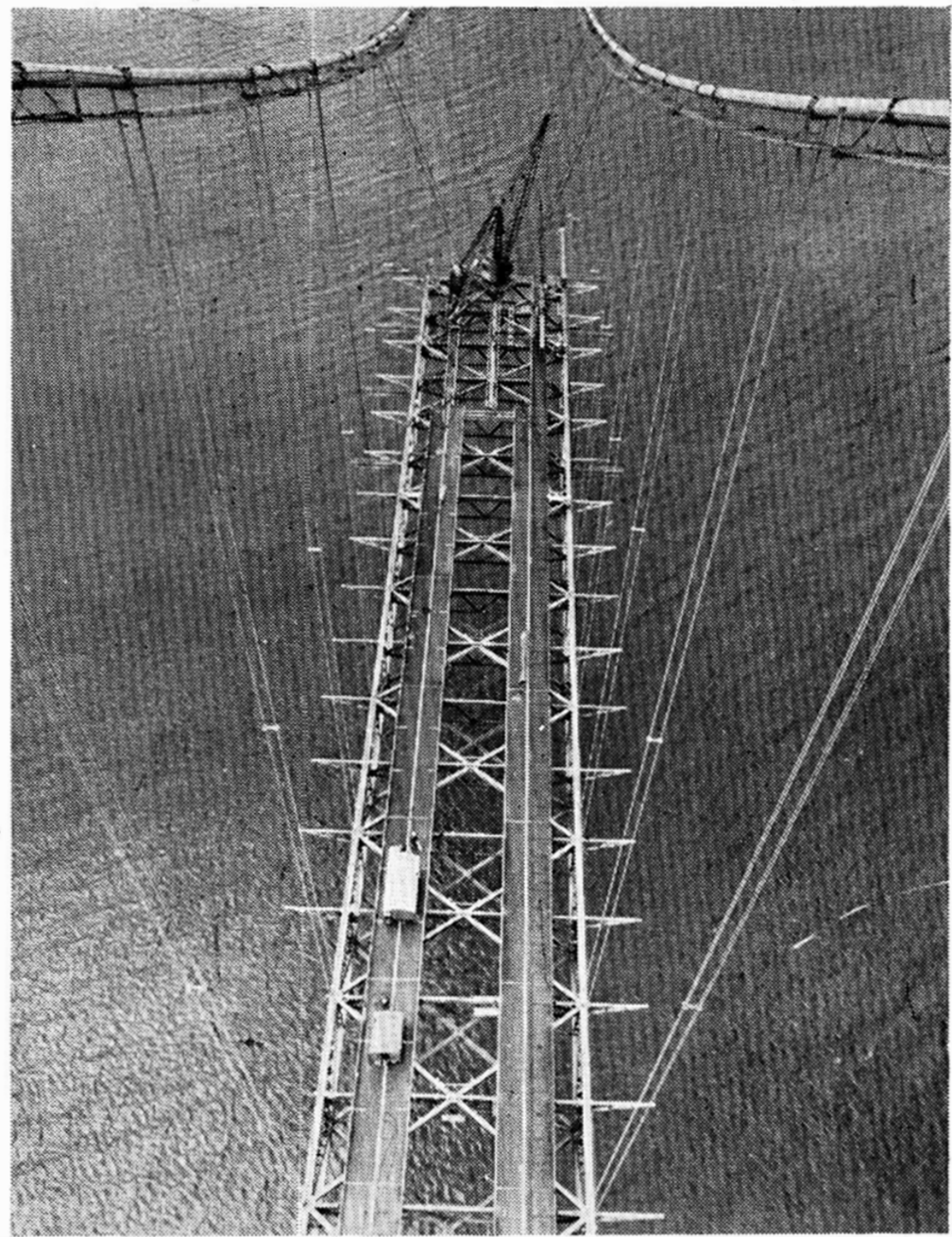

Fig. 20: ERECTION OF DECK STEELWORK IN MAIN SPAN, LEAVING OUT CENTRE PANELS OF ROADWAY UNTIL AFTER JUNCTION AT MID-SPAN 
101. This gave rise to two other questions: firstly, whether the roughness of a fairly high real wind-he was referring to the minimum natural turbulent of the real wind-was enough to prevent the vortex shedding becoming regular in structures of that type. There seemed to be some evidence for this.

102. In addition, most structures had a fairly small aspect ratio and did not approach an infinitely long cylinder. He did not know whether Roshko's experiments were done with a very long cylinder or one which had an aspect ratio of less than 20.

103. It seemed difficult to reconcile the French Government rule, where the side suction did not exceed 0.8 , with the Swiss rules where the side suction went up to 2.0 or higher, more than twice the minimum given by the French Government. That could make a greater difference to the stresses in large diameter cylinders, cooling towers, and such structures, than an increase or decrease of the same amount in the drag.

104. Information on the point might be obtained by observing existing cooling towers, since they were in the high Reynolds number range and from the top a vortex could often be seen on either side filled with steam or condensed vapour from the tower. The vortex well could therefore be observed, probably linked with the invisible vortex on either side. If there was a great deal of vortex shedding on either side the visible vortex would give some indication of what was happening.

105. Mr Wills had observed cooling towers in high wind, but apart from the action of the wind, the action seemed to be steady. Useful information might be provided from observations of cooling towers in much stronger winds.

106. Mr Wills later made the following comments in writing. Was it a correct conclusion from the information in the Paper and the film that periodic vortex shedding may take place although the structure was rigid and unable to respond appreciably

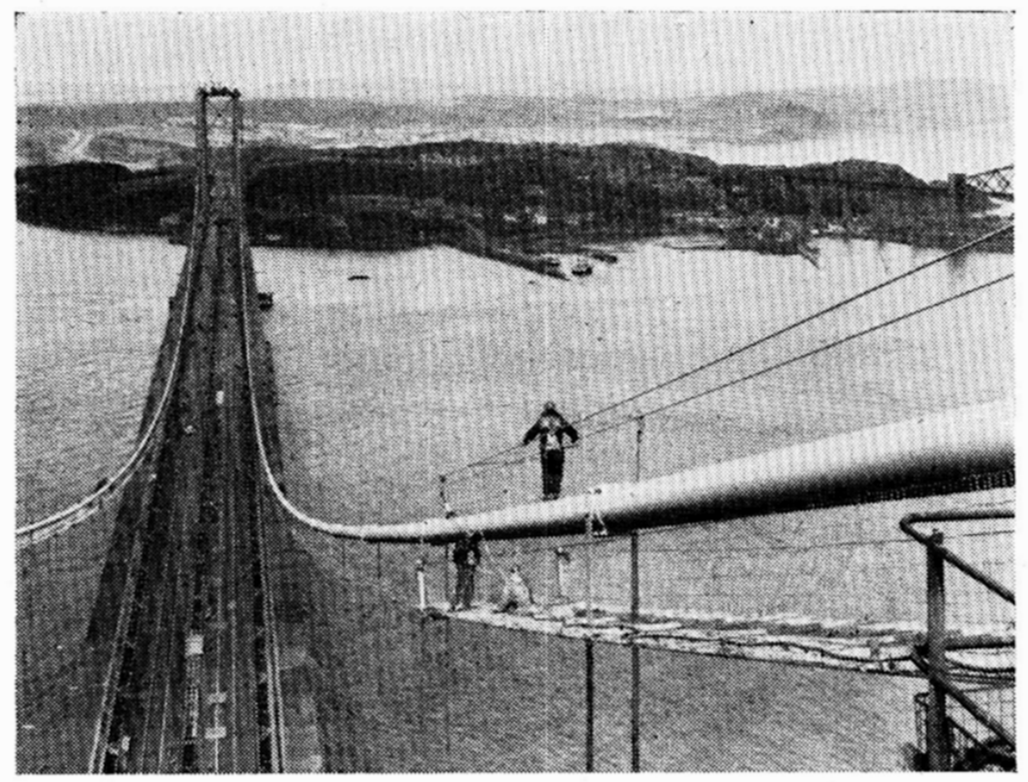

FIG. 21: REMAINDER OF DECK STEELWORK ERECTED AFTER JUNCTION AT MID-SPAN 
to the varying aerodynamic forces, but that in the case of galloping or of coupled oscillations, appreciable movement of the object was essential?

107. Of the twelve chimneys in Fig. 11, two out of the three which were reported to be unstable had Reynolds numbers in the range defined in the Paper as aperiodic, and one was slightly higher in the supercritical range. Of nine chimneys which showed no instability, only one had Reynolds number less than the super-critical range, leaving eight in the super-critical range which showed no instability.

108. The one chimney in the super-critical range showing instability may be another example of the type of vibration shown by the two in the aperiodic range and not therefore by itself a confirmation of a greater tendency to instability at Reynolds numbers above $3.5 \times 10^{\circ}$.

109. Were there any other known examples of full scale instability due to vortex shedding in the super-critical range? It was possible that if real structures did not show periodic vortex shedding in the super-critical range they might not show either the high side suction which Roshko's wind tunnel experiments report. If aspect ratio made an important difference to this, the measurements now planned to be made on a cooling tower at high Reynolds number would not settle the question for structures of high aspect ratio. Were any observations or experiments known or planned which would do this?

Mr D. J. W. Richards (Central Electricity Research Laboratories), speaking also for his colleagues Mr R. A. Scriven and Mr D. A. Davis, congratulated the Authors on a fine and comprehensive Paper.

111. They were particularly interested in the behaviour of overhead lines where both the vortex and galloping type excitations could give rise to oscillations: The natural frequencies of the fundamental modes of oscillation of overhead lines varied from $1 / 5$ to $1 / 16 \mathrm{c} / \mathrm{s}$, whereas the frequencies associated with the vortex excitation phenomenon varied from 5 to $100 \mathrm{c} / \mathrm{s}$. Consequently, unlike stacks, Aeolian vibrations of overhead lines due to vortex excitation had occurred in modes of the 30th and even the 100th harmonics.

112. The galloping of single conductors, discussed in $\S 55$ of the Paper and reference 18, had been associated with large diameter multi-strand conductors such as used on the Severn Crossing. Unlike Aeolian vibrations, that phenomenon occurred in the pseudo-fundamental and first harmonic modes. The essential difference between these two phenomena was that the frequency of the oscillation was controlled by the vortex shedding in the former and by the natural frequency of the conductor span in the latter.

113. The galloping phenomenon such as occurred on the Severn Crossing was very dependent on the wind speed or Reynolds number. During wind tunnel investigations it had been possible to maintain Reynolds number similarity between the model and full scale tests. However, it appeared likely that the maintenance of Reynolds number similarity in future wind tunnel investigations would not always be possible. For example, the study of the vortex excitation due to the flow around hyperbolic cooling tower shells would involve Reynolds numbers of the order $10^{8}$. Could the Authors give any guidance as to the correlation between model and full scale magnitudes of the vortex excitation in association with the frequency variation shown in Fig. 2 of the Paper?

114. With regard to gust induced oscillations, it had been shown in reference 18 that the amplitude of oscillations was enhanced if the components of the turbulence were correlated in such a manner that the rates of change of lift force with wind speed and yaw angle of the Severn crossing conductor were additive. Despite the presence of two mechanisms, the drag instability and the correlated gust structure, large amplitude galloping occurred on only eight of 32 occasions during which wind conditions were appropriate. This sensitivity of the galloping phenomenon to wind structure had also been found in the case of sub-conductor galloping of twin and quad 
bundled conductors for which instabilities of the den Hartog type occurred ( $\$ 10$ ). In relation to the latter, would the Authors enlarge upon $\S 18$ which referred to the behaviour of two cylinders which were vertically and horizontally mounted?

115. The wind tunnel tests which had been carried out on stranded conductors were vital in providing a basis for the understanding of the galloping phenomenon. The Paper indicated the necessity and great value of wind tunnel tests as part of the design of any large structure subjected to natural winds.

Mr R. J. Steffens (Senior Experimental Officer, Building Research Station) said that he did not speak as an expert on wind effects on buildings or structures generally. His particular interest was in man-made vibrations and their effect on structures, and in that context he had several comments to make.

117. First, on the subject of structural damping, it appeared from Table 1 of the Paper that the logarithmic decrement of damping for the cases given was usually of the order of 0.06 . But those data referred specifically to towers and stacks. He wondered whether the Authors had any information about the value of the logarithmic decrement of damping for structures generally and buildings in particular.

118. His second point related to the natural periods, or frequencies, of structures. Was there any simple formula whereby the natural period of, for example, a concrete chimney could be calculated without reference to calculations by more laborious means? At the moment it would seem that the calculation of the natural period, which was a factor of great importance, was a very laborious and complicated business.

119. A paper by Rumman ${ }^{55}$ had appeared recently, which Dr Flint might not have seen. It referred to a computer technique for calculating the natural periods of the four fundamental modes of vibration of tall reinforced-concrete chimneys with steel linings.

120. So far as buildings themselves were concerned, there were various empirical formulae in existence. Examples of these were the formulae given by the American Society of Civil Engineers, by Housner, and others. Using these formulae, the natural frequency could be determined from the 'geometry' of the building, i.e. the height and base dimensions, or even the number of storeys. The height of the building appeared to be the most significant factor. For a building $150 \mathrm{ft}$ high, for example, the natural period was likely to be of the order of 1 second, whereas for a height of $500 \mathrm{ft}$ the period was likely to be 3-4 s. The question was whether similar simple formulae existed for stacks and chimneys.

121. Mr Steffens' third point was in connexion with the effect of vibration on people. The question of structural motion in a high wind had been referred to, and the Chairman had said that the sway of about $7 \mathrm{ft} 6 \mathrm{in}$. on a free-standing tower of the Forth Road Bridge had produced a feeling of seasickness in the workmen. $\mathrm{Mr}$ Steffens was not surprised by this, if the natural period was of the order of $4 \mathrm{~s}$. The effect of vibration on people depended mainly on two quantities - the frequency and amplitude of the vibration. It was possible in the case of tall buildings that the combination of frequency and amplitude of oscillation might well be such as to produce a feeling of discomfort. Bearing in mind the current tendency towards increasingly taller buildings, including offices and blocks of flats, that seemed to him to be an important question to which attention ought to be given, but it was unfortunately often overlooked.

122. The new Museum telephone exchange was a case in point. It was not an occupied building in the true sense of the term, but it had a restaurant at high level, so that the point was material. The figures which he had in mind for that building were that the vibration amplitude in a wind of about $70 \mathrm{miles} / \mathrm{h}$ was likely to be about 6 in. and the natural period of the building was likely to be about $3 \mathrm{~s}$. If those figures were correct, it would appear from all the evidence about human sensitivity 
to vibration ${ }^{56}$ that when a high wind was blowing, people in the restaurant could be very uncomfortable.

123. The structural engineer would, he thought, be very grateful if some guidance could be given on this problem. Was there a formula, for instance, whereby the probable amplitude of oscillation for a particular building or structure in a given wind condition could be calculated? If such a formula were available, and the natural frequency could be established from the height and base dimensions, one would have something to work on, and could say whether the resulting vibration might be intolerable to the occupants.

Mr D. C. Tobutt (British Reinforced Concrete Engineering Co. Ltd), who said that he had in mind reinforced concrete chimneys, asked how the logarithmic decrement could be calculated in such a case, and whether further progress had been made with the theoretical investigation. He understood from $\mathrm{Mr}$ Scruton that it was still largely empirical. As structural engineers they wanted to know how wind velocity would affect the vibration of chimneys. It was a little baffling that the logarithmic decrement could not be known precisely. $V / N D$, the other parameter, could be calculated, and a rough idea formed of whether the chimney was stable by calculating both parameters and seeing whether the point fell within the shaded, i.e. unstable, region of the graph.

125. They had calculated the period of natural vibration from formulae presented by Taylor and Turner, ${ }^{57}$ and they had prepared a computer programme, which worked on the Sirius machine, for calculating the fundamental, but they were still in doubt about the logarithmic decrement.

Mr P. S. A. Berridge (Consultant, Messrs Donovan H. Lee \& Partners) noted that the film appeared to show that turbulence in water flowing past a circular obstruction was greater on the downstream side. This shouid not be confused with scour at a bridge pier. At a pier, circular in plan, scour was greater on the upstream side and in the event of the pier being scoured out, it would topple over against the current.

127. He also drew attention to the diversity in practice in the programmes for the erection of the stiffening girders in long-span suspension bridges. For instance, at the Tamar Bridge erection had commenced in the middle of the span and been continued in equally balanced stages towards the main towers; but at the Forth the girders had grown outwards from the towers until they had met at the centre of the span. Was there any particular merit in adopting the one or the other method?

Mr I, P. Haigh (Research Engineer, Sir Alexander Gibb \& Partners) said that the Chairman had mentioned the distinction between statics and dynamics. The knowledge of dynamics amongst civil engineers as a whole was limited, but those described as bachelors of science were fully conversant with statics and could have an easier conscience about dynamics; masters of arts had no excuse.

129. Characteristically, therefore, at last summer's conference on wind effects on buildings at the National Physical Laboratory, the civil engineers present, other than those directly concerned with this field of work, were unable to play a prominent part. One of them, however, described an unusual occurrence of fatigue failure in the handrailing of a bridge.

130. It might therefore be of some interest to the Authors to learn of a similar incident which had occurred since the conference, on a dam now under construction on the Nile.

131. Starter bars, $1 \frac{1}{2}$ in. in diameter, had been left deliberately projecting some $6 \mathrm{ft}$ above a construction joint that was to be submerged during the wet season. When the floods subsided after the steel had been exposed for some months in a fastflowing stream, it was found that some of the bars had fractured and were missing completely, to the dismay of the engineers on site. Faults in the steel and corrosion 
were only dismissed as possible explanations when simple calculations showed that the Strouhal frequency of vortices thrown off by the bars almost exactly corresponded to their natural frequency as cantilevers.

Professor A. J. S. Pippard (Past President) said he was pleased that the Institution was beginning to show an interest in one of the most important aspects of structural loading. His object in speaking was to express his thanks to the Authors for a very important Paper and to add a short historical note.

133. The phenomena dealt with in the Paper were mostly those which the Authors described as self-excited, but reference was made to proximity effects and, in particular, to one of these which the Authors called 'buffeting'.

134. In 1930 a terrible accident occurred to a low-wing monoplane at Meopham in Kent, in which a party coming from France was killed. It was a complete breakup in the air, and the wreckage was spread over about two miles. He was one of the few survivors of the committee which investigated the accident.

135. The Inspector of Accidents at the Air Ministry reported that the evidence available was such that he had been unable to arrive at a definite conclusion as to the cause of the accident, and his interim report stated that careful examination had revealed no defects in structure and no signs of faulty or inferior material.

136. It was known that oscillations called flutter could occur in high speed flight, and it was believed that this might be the explanation of the accident. The possibility was carefully investigated at the National Physical Laboratory by means of a model which proved that tail flutter could not occur within the speed range of the aeroplane. However, another possible cause of tail oscillation emerged, provisionally called buffeting, which occurred at incidences near the stalling attitude.

137. The National Physical Laboratory investigated this by a semi-elastic model and found that eddies shed from the wing caused the tail to oscillate violently at relatively low speeds. No doubt remained that this was the cause of the accident.

138. Professor Pippard was interested to note that the term had survived, as undoubtedly had the phenomenon which gave rise to it, and it occurred to him that this interference effect due to the shedding of eddies from some obstruction ahead of the structure was a matter of great importance. An example of it was shown in the excellent film they had seen, but it could also occur in a group of high chimneys, such as those of power stations. He felt that this particular phenomenon might well cause serious trouble in certain arrangements of such stacks.

Mr H. C. Adams (Ministry of Public Building and Works) said that at the Ministry he had been concerned with the design of the Post Office tower at the Museum telephone exchange. As would be noted, they had had the advice and assistance of Mr Scruton in designing it.

140. It was true that the Paper produced a curve showing an amplitude of about 3 in. at $75 \mathrm{mile} / \mathrm{h}$, and he would not suggest that that was not correct. He would point out, however, that $\mathrm{Mr}$ Scruton's wind tunnel was carefully designed to produce an absolutely uniform wind velocity, which did not occur in the open in practice.

141. In the case of a tall building, such as the Museum Tower, a speed of about $65 \mathrm{mile} / \mathrm{h}$ at ground level would produce a corresponding speed at the top (600 ft) of between 90 and $95 \mathrm{mile} / \mathrm{h}$. That meant that the critical velocity was occurring at only one place at any one moment, something for which engineers could be grateful, because it gave them something in hand. It could mean, he thought, that they had in practice a rather better condition than the wind tunnel would show. He felt that the wind tunnel test, in fact, tended to be pessimistic, which was perhaps a good thing.

142. In calculating natural frequency, it might be economical, if one had a large number of similar or approximately similar chimneys, to use a computer programme, but otherwise he would have thought that the Stodola analysis was not too laborious 
and was very satisfactory where there were large variations in mass or inertia along the length.

Mr B. J. Vickery (University of Sidney) said that he had some information regarding the proximity effects mentioned in $\$ \S 17$ and 18 of the Paper. It concerned some observations made both on a model and at full scale on a group of four chimney stacks.

144. The chimney stacks were $190 \mathrm{ft}$ high, although the base was $150 \mathrm{ft}$ above the ground, as they were situated on the top of a power station. The stacks were $13 \mathrm{ft}$ in diameter and there were four in line at $54 \mathrm{ft}$ centres. The natural frequency of the stacks was about $1 \mathrm{c} / \mathrm{s}$, the predicted critical wind speed was $40-45 \mathrm{mile} / \mathrm{h}$, and the logarithmic decrement as measured by rocket tests was 0.06 . The chimneys were of welded steel and were lined with plastic refractory material, which added little to the damping. The major part of the damping could be attributed to the fact that the chimneys were mounted on the roof of a power station and the roof was of riveted steel contruction. The chimneys were far from having fixed bases, and there was a fair amount of energy moving into the building and being dissipated in the riveted steel work.

145. In the terminology of the Paper, the non-dimensional damping was 8 . As to the full scale observations, with the wind blowing normally to the line of the stacks, amplitudes of up to about 12 in. peak to peak were registered, whereas with the wind coming down the line or within $30^{\circ}$ of it, the maximum amplitude increased as one moved down from the first stack to the last in the line. In the case of stack 4 and also stack 3 a measured amplitude of about $3 \mathrm{ft}$ peak to peak was obtained. The oscillations brought about fatigue failure in the welded joints near the base of the stack, which had to be attended to before the investigation could be continued, in order to ascertain some means of stopping it.

146. Model tests ${ }^{58}$ were conducted, and for the worst case the model amplitude was about twice that observed at full scale. This discrepancy might be attributed to the effect of Reynolds number, to the non-uniformity of the wind or to a number of other factors. The comparison, however, gave some measure of the reliability of wind tunnel tests conducted at the low values of Reynolds number and applied to Reynolds numbers of the order of $10^{7}$, which was relevant for the full scale structure.

147. The addition of strakes to the stacks was one of the means tried. As $\mathrm{Mr}$ Scruton had pointed out in the Paper, those behaved well for a single stack, and for a wind normal to the line they completely eliminated vibration in the model. They were not successful, however, in eliminating the vibrations in the fourth stack with the wind coming down the line or very close to it. In that instance the model indicated amplitudes of the same order as those which were already occurring with the full scale structure.

148. Nothing had yet been done to solve the problem completely, but the addition of damped tie-rods or guys from the stack to the top of the power station seemed a reasonable solution.

149. Although the addition of the strakes did not improve the amplitude of oscillation, it altered the behaviour in that the strakes increased the critical wind speed from $V / N D=5$ to $V / N D=8$ to 9 . This increase in wind speed would materially reduce the frequency of occurrence of the vibrations and hence add to the fatigue life of the structure.

Mr D. A. Davis (Central Electricity Generating Board) said that he wished to ask the Authors a question about $\S 54$ of the Paper, which he thought was a depressing statement from the point of view of galloping-excited oscillations. If a gallopingexcited oscillation was present, presumably one of two things could be done-either eliminate the aerodynamic excitation or increase the structural damping. The Authors stated that the latter did not help very much, but surely increased structural 
damping added to the damping due to aerodynamic drag and thereby reduced the margin of instability. If this were not effective in eliminating the galloping oscillation at the particular speed at which it was occurring, as the Authors said, it might increase the critical speed. The slope of the lift incidence curve might well be Reynolds number-dependent, however, in which case an increase in the critical speed might well help to suppress the oscillation. Would the Authors comment?

The following contributions were received in writing:

Dr D. I. H. Barr (Senior Lecturer, Department of Civil Engineering, University of Strathclyde) wrote that he wished to make some comments and enquiries regarding the more elementary aspects of this interesting Paper. Included in the notation and used throughout the paper were

Reynolds number, $R$ (presumably $V D / v$ )

Strouhal number, $S=f D / V$

Reduced velocity, $V r=V / N D$

where $f$ is the frequency of vortex shedding

$D$ is a typical length

$V$ is the wind velocity

$N$ is the natural frequency of a structure

$\nu$ is the kinematic viscosity of the fluid flowing.

152. The Strouhal number contained two flow characteristics (velocity and frequency), in common with other definition groups, and while it might be an indication of kinematic similarity, it was not a criterion of dynamic similarity. This was not to say that kinematic and dynamic similarity of a fluid system could be effectively separated, but merely that the Strouhal and Reynolds numbers should be explicitly differentiated, as indeed the Authors had implicitly differentiated them. An intermittent discussion on the place of the Strouhal number had appeared in engineering proceedings and journals in the past few years. ${ }^{59-67}$ While Dr Barr agreed with certain of the protagonists that the various types of group should be classified as an aid to understanding, he did not think that the Strouhal number should be placed with the Reynolds, Froude and similar dynamic criteria. Having made considerable use of the Strouhal number, the Authors were well placed to comment on this.

153. Other groups of the same general nature as the Strouhal number could be written for any other flow characteristic (apart from velocity) each containing that characteristic and velocity, for example:

$\begin{array}{ll}T V / D & \text { 'Time number' } \\ Q / D^{2} V & \text { 'Flow number' } \\ F / \rho D^{2} V^{2} & \text { 'Force number' } \\ \operatorname{Pr} / \rho V^{2} & \text { 'Pressure number' } \\ P o / \rho D^{2} V^{3} & \text { 'Power number' }\end{array}$

where $T$ is a time interval

$\mathcal{Q}$ is a flow

$F$ is a force

$\operatorname{Pr}$ is a pressure

$P o$ is a power

$\rho$ is the fluid density.

154. These were examples of the many such groups. One wondered why two (Strouhal number and Newton force group) had been named after investigators, a further one was commonly called the Power group, while the others seemed to be unnamed. 
155. Dr Barr thought it would be useful if the Authors discussed briefly the practical limitations, difficulties, and procedures of model testing. A first approach to the design of a model test might be to choose a linear scale to fit the wind tunnel available. With a prototype maximum velocity in mind and using air (of about the same density and kinematic viscosity) the model velocity would be increased in inverse proportion to the decrease in linear size to maintain the same Reynolds number. The required frequency of the model structure would then emerge from the Strouhal number criterion, or equally from the 'time number' using the periods of oscillation. This stage would involve the characteristics of the model structure and might be regarded as the obtaining of partial kinematic similarity of the combined fluidstructural system, without ensuring correspondence in an amplitude of the structural movements. It seemed possible that useful results might be obtained even at this stage. The next stage would be to seek to link the two systems dynamically by making the model such that the force necessary to cause some chosen relative deflexion in the model be scaled according to the force number, $F / \rho D^{2} V^{2}$, while retaining the desired frequency of oscillation.

156. The above represented perhaps a rather over-simplified approach to the design of model tests. On the other hand, one avoided the introduction of groups and concepts which principally aided the analytical approach. The use of reduced wind velocity, where the name had connotations of the fluid system but where a change in the structural properties changed the value of the group-other things remaining equal-might well have advantages, but also seemed to have hazards, and $V_{\mathrm{r}}=V / N D=1 / S$, as given in $\S 20$, could be ambiguous regarding similarity in the fluid flow.

157. Dr Barr suggested that the Authors might comment on the choice of materials for the structural models and say whether water tunnels or wind tunnels with compressed air had any application to this type of work.

Dr T. A. Wyatt (Imperial College) wrote to draw attention to one factor which acted to reduce the likelihood of instability of the galloping type. Referring to polygonal section members ( $\$ 51-54$ ), the Authors stated that many common structural sections were liable to galloping excitation, but pointed out that this applied only for a limited range of wind direction in each case.

159. During oscillation of an elastic member, any element could be regarded as in equilibrium between inertia forces and the elastic restoring forces. The actual periodic aerodynamic forces were generally relatively small and insufficient to force any significant change in the mode. Galloping would therefore only occur if the critical range of wind direction included the direction of motion in the natural mode.

160. The angle section with equal legs was commonly used in structures exposed to the wind, and plotting the basic force coefficients showed ranges over which the 'negative lift slope' criterion of $\S 10$ applied. The minor axis bending direction was near the limit of such a range and might be weakly excited, but in most practical cases the end connexions were not symmetric with respect to the angle. In this case the motion would be complex, but the tendency was probably toward stability.

161. Dr Wyatt had been interested in wind-excited oscillations for many years, but had not seen a single angle oscillating, presumably for the reasons just mentioned. The only example he had seen of a structural section in motion which might be of that type were double angles spaced apart back to back, with the wind incident almost directly through the gap. For this condition, tests showed a sharp decrease of lift when the angle of incidence reached a few degrees, the new value persisting until the incidence was reversed to a similar angle in the opposite direction. This presumably corresponded to two possible flow patterns through the gap, and in contrast to the more common form of galloping, could excite only a very limited amplitude. The observed oscillations were probably of this type, as they persisted 
over a wide range of wind speed, but were not otherwise distinguishable from vortex shedding.

The Authors, in reply, thanked Mr Gilbert Roberts and the Chairman for their comments on the progress of the Forth Road Bridge. They were particularly gratified to hear from the Chairman that the erection procedure which had been suggested by the National Physical Laboratory had been successful.

156. The Chairman had remarked on the fact that the Forth Bridge towers had remained stable under high wind speeds. This would be predicted by the curves in Fig. 4 of the Paper. It would be seen that for a rectangular shape the aerodynamic excitation built up very rapidly at a certain reduced wind speed, but that at higher wind speeds for the same structure, the excitation began to reduce, and not until a speed of about five times that initially causing excitation should the serious oscillations recur, either of galloping nature in the fundamental mode, or vortex excited in the first harmonic mode. As the Forth Bridge first oscillated at $25 \mathrm{mile} / \mathrm{h}$, it might require a wind speed of at least $125 \mathrm{mile} / \mathrm{h}$ to cause it to oscillate again. This would exceed the practical range of steady winds.

157. Mr Wills had asked a question about vortex shedding and had inquired what was the extent of the available information on this phenomenon. Most of the available information referred to the sub-critical range of Reynolds number, less than $3 \times 10^{5}$. They knew that vortex shedding was aperiodic above a certain value. It was only recently that Roshko's experiments suggested that there was a return to periodicity in the air flow at Reynolds numbers of about $3 \times 10^{6}$. Roshko's tests only went to about $10^{7}$, and that was about the highest reached in wind tunnel experiments in the measurement of vortex frequency. Unfortunately, there had not been any measurements of aerodynamic excitation at these very high values of the Reynolds number and this was a suggestion for further research in the Paper.

158. With regard to Mr Wills' comments on the incidence of instability of stacks in the higher range of Reynolds number $\left(\operatorname{Re}>3 \times 10^{6}\right)$ as given in Fig. 11, it should be noted that for five of the stacks cited, the calculated critical wind speed was well above the range of natural winds, leaving only four with critical speeds within the range. However, the Authors agreed that the evidence of Fig. 11 of the Paper did not indicate greater tendency for stacks to oscillate at Reynolds numbers above $3.5 \times 10^{8}$. The Authors could not give details of other stacks which had oscillated but quoted den Hartog ${ }^{68}$... . recent observations of large industrial smoke stacks vibrating violently at the correct Strouhal frequency with Reynolds numbers as high as $10^{7}$. The NPL plan experiments to measure vortex shedding frequencies and the vortex excitation for cylinders of various aspect ratios at the high Reynolds numbers obtainable in a wind tunnel pressurized to 25 atmospheres, but even with this degree of pressurization it would be difficult to achieve Reynolds numbers in excess of $3 \times 10^{6}$.

159. Periodic vortex-shedding takes place from a rigid structure, but, of course, there is no energy exchange between windstream and structure until there is finite movement of the structure. Similarly the conditions for instability in galloping or coupled oscillations obtain for infinitely small amplitudes, and for linear systems 'appreciable' amplitudes are not necessary for the instability to develop. However, for all three causes of instability mentioned above, finite amplitudes may be required because of non-linearities, and particularly because of non-linearity of the structural damping.

160. Mr Wills commented on the discrepancy between the values for 'side suction' on circular cylinders given by the French $\left(C_{\mathrm{p}}=-0.8\right)$ and the Swiss Codes $\left(C_{\mathrm{D}}=-2 \cdot 6\right)$. Roshko's experiments gave $C_{\mathrm{p}}=-2 \cdot 0$ at the high values of Reynolds number and referred to infinitely long cylinders. The maximum suction (and the vortex excitation) reduce as the aspect ratio of the cylinder decreases but the Authors 
had no evidence to suggest that it would fall as low as 0.8 except perhaps for cylindrical tanks of extremely low aspect ratio.

161. With regard to observations of pressures on existing cooling towers and extreme suctions, which Mr Wills had mentioned, some work on that very problem was proposed by the Central Electricity Generating Board. The Authors did not know whether or not the Board had started any measurements, but believed that instrumentation was being prepared to make those observations.

162. Mr Richards had raised a question concerning two cylinders side by side in line or across the wind. Useful information had been given by Mr Vickery on the buffeting effects of stacks in line. The Authors did not wish to enlarge further on the excitation of two stacks in close proximity. The statement made in $\S 18$ was introduced into the Paper to bring proximity effects to the attention of engineers; the existing information was scanty.

163. With regard to the variation of the value of vortex excitation with the frequency variation shown in Fig. 2 of the Paper, discussed by Mr Richards, no precise information was at the time available. It appeared certain that the highest excitations occurred in the sub-critical range of Reynolds number, but it was not known to what extent the excitation recovered on passing from the range of Reynolds numbers for the aperiodic of vortex shedding to the range where Roshko again found periodicity in the wake. In the practical application it should be noted that cylinders of finite length were involved and that the excitation was sensitive to the aspect ratio and to other factors such as the turbulence of the approaching air stream.

164. With regard to damping data, Mr Steffens had asked whether or not there were any data for buildings in general. The Authors had had great difficulty in finding any results at all to put into the Paper, most available evidence relating to steel structures. The Authors could not quote any other results but believed that measurements were being made on tall buildings on the west coast of the United States. One of the aims of the Paper was to encourage the undertaking of fairly simple but valuable tests on completed structures to assist in the accumulation of damping data.

165. Regarding the calculation of natural frequencies and their prediction by empirical rules, the Authors knew of no simple answer. The formula due to Housner, referred to by Mr Steffens, had been found unreliable and earthquake engineers could not agree on such empiricism. Reference had been made in the discussion to several methods in common use for calculation some of which were amenable to computer techniques which were particularly valuable when dealing with the higher modes of structures such as guyed masts. Programmes were available which give satisfactory results.

166. A thin-walled cylindrical cantilever of uniform section would have natural frequencies proportional to $D / H^{2}$ (in the notation of Fig. 11). The Authors had plotted in Fig. 22 the calculated fundamental frequencies for the stacks mentioned in the Paper. Owing to the influence of such factors as taper and thickness variations of stack and lining, the ratio $D / H^{2}$ was not a sufficient criterion alone to provide an accurate guide and the wide variations evident from the graph were to be expected. However, the results might be useful as providing an indication of the order of magnitude of the frequency.

167. With regard to the effect of vibration on people, the Authors considered that at heights of $600 \mathrm{ft}$ and more there was a possibility of wind noise being a more serious problem than vibration. The acceptance by human beings of vibration had been studied by the aeronautical engineers in some detail, but possibly at frequencies which were out of the range with which Mr Steffens was concerned.

168. The final question by $\mathrm{Mr}$ Steffens was in regard to the prediction of the amplitude and frequency of buildings excited by wind. Unfortunately there was neither sufficient aerodynamic information or information on structural damping to enable the amplitudes to be predicted. It would seem that the same considerations 
applied to calculation of amplitudes of stack oscillations which were mentioned by Mr Tobutt.

169. To calculate accurately the damping of a structure was not feasible at the present time. Mr Tobutt had raised this question in relation to reinforced concrete chimneys. In such a structure internal hysteresis losses would vary with the mix used by the contractor on the site, the type and quantity of reinforcement and the stress range in all parts of the material. Moreover the nature of the foundations could significantly influence the total damping. It was, however, noticeable that for a given class of structure, the damping was reasonably constant, and it was probably satisfactory to measure the damping for a given class and to use that in calculation.

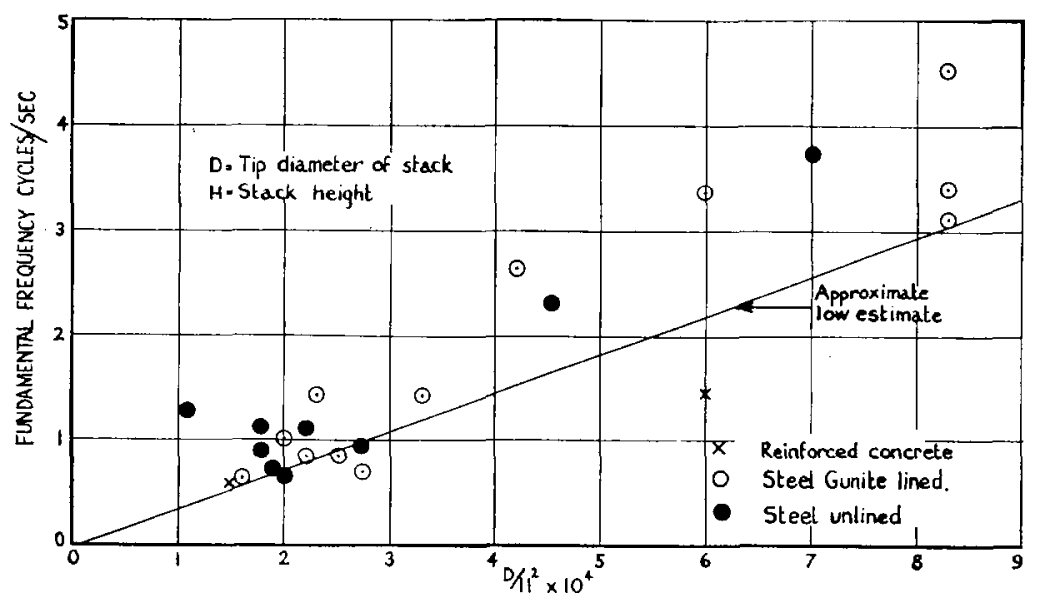

Fig. 22: ESTIMATED FREQUENCIES FOR STACKS

170. In the particular case of a bolted steel structure or one with controlledfriction joints a conservative estimate of the damping could be made in much the same way as for the Crystal Palace tower. That was useful if one found that, in the condition before joint slip, the damping was insufficient to ensure stability. It was found that the damping increased very considerably with amplitude in such structures and this fact possibly accounted for the lack of catastrophic failures due to vibration. The difficulties of predicting structural damping emphasized the need for further research.

171. With regard to Mr Berridge's remarks about scouring of bridge piers, it was not entirely unexpected that scouring would occur near the upstream side of the pier, for there the velocity round the pier would be greatest.

172. Mr Berridge had mentioned varying practice in the erection of suspension bridges. The question of erection sequence was involved and outside the scope of the present Paper. In essence the choice depended on the need to restrict the lifted load to a small percentage of the main cable tension at any time and to limit the sway of the tower or movement of the saddles. The Tamar Bridge, with its light cables and sidespans overland, differed in both respects from the Forth Bridge. Contractors' preference for certain handling techniques greatly influenced the scheme and one of the Authors had assisted in devising a tender scheme for the Tamar 
Bridge in which deck erection proceeded mainly from the towers inwards, which was as viable as the alternative adopted.

173. Mr Haigh had referred to the aluminium handrailing which had vibrated on the bridge described by Mr Clark at the 1964 NPL Conference on Wind effects on buildings and structures, and had also told them about the starter bars with their coincidence between eddy frequency and the natural frequency of vibration. The Authors wondered whether, in calculating the natural frequency of vibration, account had been taken of the virtual inertia of the water, which might be quite considerable. This inertia could be omitted in the case of air, but its effect in calculating the frequencies in water might be important.

174. Professor Pippard had revived memories about the Meopham disaster and the survival of the term 'buffeting'. It was perhaps not surprising that the term had survived since one of the Authors had been concerned with the tests relating to the Meopham disaster, and had been in at the 'birth' of the use of the term to describe the aircraft phenomenon and had therefore used it for similar problems in civil engineering.

175. The Authors were not sure that Mr Adams would get all the relief that he expected from the vertical wind gradient. Admittedly, the wind varied with the height, and one might expect from that that the vortex frequency would vary and, hence, that there would be no correlation between the exciting forces along the height of the tower. But it had been found with tapered stacks, where again one might expect the same effect, that the vortex frequency seemed to lock into a certain frequency for a considerable portion of the height of the stack. Therefore, the same might happen with a velocity gradient. That was an aspect which was due for research at the National Physical Laboratory.

176. Mr Vickery's experience with the four stacks in line was very interesting. The Authors would not expect the strakes to be effective in the case of buffeting since the strakes themselves did not prevent periodic vortices being discharged into the wake. They were effective as stabilizers because they destroyed the correlation along the height of the stacks.

177. Mr Davis had found the statement in $\S 54$ about the prevention of the galloping type oscillations to be rather depressing. The Authors had not meant it to read as such. With regard to the complete prevention of galloping oscillation, they did not know of anything effective except the selection of a suitable aerodynamic shape. Even so, ice accretion could still create the problem. An increase in structural damping would increase the critical wind speed by a certain amount, but in general not much relief would be expected from the Reynolds number dependence of the forces, especially when there was ice formation which tended to build a shape for which the forces would not be so dependent on Reynolds number.

178. Dr Barr's comments touched on the fundamental principles of aeroelastic model testing. Space was not available in the Paper to describe the techniques employed at NPL, and they could only be briefly dealt with. The requirements for dynamic similarity of two elastic systems in a fluid flow could be argued on the usual methods of dimensional analysis. For civil engineering structures the relevant physical quantities influencing the aeroelastic behaviour are

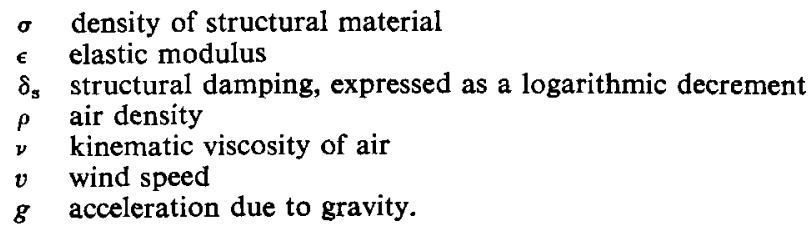

179. For similarity the model and prototype should have the same geometric 
shape, and the same density stiffness and damping distribution; and the following non-dimensional parameters should have the same values
(i) $\sigma / p$
density parameter
(ii) $E / p V^{2}$
elasticity parameter
(iii) $\delta_{\mathrm{s}}$ damping parameter
(iv) $g L / V^{2}$ gravitational parameter (c.f. Froude number)
(v) $V L / v$ viscosity parameter (Reynolds number).

180. It can be noted that, because of the dependence of $N$ on $\sqrt{ } E / \sigma$ either (i) or (ii) can be replaced by the reduced velocity $V / N D$, provided similar values of all the other parameters are maintained. The substitution of $V / N D$ for $E / p V^{2}$ is often useful because it permits departures from the replica form of model construction.

181. With models of reduced linear scale tested in the usual atmospheric wind tunnels it is evidently not possible to satisfy (ii), (iv), and (v) at the same time and some relaxation of the requirements is necessary. Almost invariably Reynolds number is the parameter discarded, although it is known that the air flow pattern, the Strouhal number, and the wind forces, are all dependent on Reynolds number for structures of circular or rounded sections. In such cases some reservations have to be placed on the extrapolation of the model results to full scale. The same reservations need not be applied to structures of bluff sharp-edged shapes which are not subject to the same dependence on Reynolds number.

182. The art in the design of aeroelastic models to give reliable predictions of instability lies in the knowledge of the extent to which the similarity requirements given above can be modified or relaxed. For example, in considerations of models of stacks it is permissible to ignore the gravitational parameter, because compressive stresses do not significantly influence the oscillation frequencies; on the other hand it is evident that this is an all important parameter in considerations of suspension bridge oscillations.

183. It would be noted that Strouhal number, Newton force number etc., were not mentioned in the above list of similarity requirements. These quantities are dependent on one or more of the independent similarity parameters, for example

$$
S=f \text { (shape, } R e \text { ). }
$$

184. The Authors agreed that there was no consistency in the way in which the various dimensionless groups were described, and they would suggest that a terminology using names descriptive of the important physical quantity was to be preferred to the use of personal names.

185. Reference had already been made to the use of pressurized wind tunnels for aeroelastic investigations; at the NPL it was hoped to make increased use of such a facility. Water tunnels could be used in some applications but the advantages gained because of the reduced kinematic viscosity as compared with air was usually more than offset by the lower speeds and the smaller dimensions of water tunnels. There was also the further disadvantage of the requirement to construct an intricate model of very small scale in a material many times heavier than the material of the prototype, as prescribed by the density parameter (i).

186. Dr T. A. Wyatt had not seen an angle structural section oscillating due to wind. Neither had the Authors, but undoubtedly there were conditions when an angle section would oscillate, particularly if it was used in long unsupported lengths with consequent low frequencies. It seemed to be implied by Dr Wyatt that oscillation could only occur by bending about the minor axis and due to galloping type excitation. Other directions of oscillation were, however, possible at different frequencies and vibrations could also be excited by shed vortices and possibly by coupling. Thornton ${ }^{69}$ had reported oscillation in torsion, bending and coupled modes of approximately $18 \mathrm{ft}$ long members of 2 in. $\times 2$ in. $\times 0.10$ aluminium angle. Steel angle of 2 in. $\times 2$ in. $\times \frac{1}{8}$ in. section also showed bending oscillations. 


\section{REFERENCES}

55. W. S. Rumman. Vibrations of steel-lined concrete chimneys. Proc. Amer. Soc. civ. Engrs, vol. 89, no. ST5, pt 1, pp. 35-63.

56. D. Dieckmann. A study of the influence of vibration on man. Ergonomics, vol. 1 , no. 4, 1958, pp. 347-355.

57. C. P. TAYlor and L. Turner. 'Reinforced concrete chimneys'. Concrete Publications Ltd, 2nd ed. 1960.

58. B. J. VICKERY and R. D. WATKINS. 'Flow induced oscillations of cylindrical structures'. Proc. 1st Aust. Conf. on Hydraul. and Fluid Mech., 1962. Pergamon Press, London, 1963.

59. F. V. A. ENGEL. Non-dimensional groups and the model laws of dynamic similarity. The Engineer, vol. 198, no. 5154. 5 November, 1954, p. 637.

60. J. M. ZAREK. Metering pulsating flow; coefficients for sharp edged orifices. Engineering, vol. 4641, 7 January, 1955, pp. 17-19.

61. F. V. A. ENGEL. Metering pulsating flow; Strouhal number and Hodgson criterion. Engineering, vol. 179, no. 4645. 4 February, 1955, p. 133.

62. E. G. Chilton. Metering pulsating flow-significance of non-dimensional criteria. Engineering, vol. 179, no. 4655. 15 April, 1955, p. 459.

63. F. V. A. ENGEL. Strouhal number and model laws of dynamic similarity. Engineering, vol. 179, no. 4663.10 June, 1955, p. 717.

64. F. V. A. ENGel. Non-dimensional groups as criteria of process plant dynamics. The Engineer, vol. 206, no. 5357. 26 September, 1958, pp. 479484.

65. S. W. E. EARLeS and J. M. Zarek. Use of sharp-edged orifices for metering pulsating flow. Proc. Instn mech. Engrs, vol. 177, no. 37, pp. 997-1024 (incl. reply to discussion).

66. F. V. A. ENGEL. In discussion of ref. 65.

67. A. A. Putman. An extension of Engel's views on dimensional analysis. The Engineer, vol. 217, no. 5636. 31 January, 1964, pp. 215-217.

68. J. P. Den Hartog. Recent technical manifestations of Von Karman's vortex wake. Proc. Nat. Acad. Sc., vol. 40, No. 3, 1954.

69. C. P. Thornton. 'Wind tunnel tests of the aerodynamically induced vibrations of some simple structural members.' Nat. Res. Co. of Canada, Mech. Eng. Rep. MA-245, December 1962. 